\title{
Body size in Drosophila: genetic architecture, allometries and sexual dimorphism
}

\author{
VP Carreira, J Mensch and JJ Fanara \\ Departamento de Ecología Genética y Evolución, Facultad de Ciencias Exactas y Naturales, Universidad de Buenos Aires, Ciudad \\ Universitaria, Pabellón II, Buenos Aires, Argentina
}

\begin{abstract}
Even though substantial progress has been made to elucidate the physiological and environmental factors underpinning differences in body size, little is known about its genetic architecture. Furthermore, all animal species bear a specific relationship between the size of each organ and overall body size, so different body size traits should be investigated as well as their sexual dimorphism that may have an important impact on the evolution of body size. We have surveyed 191 co-isogenic lines of Drosophila melanogaster, each one of them homozygous for a single $P$-element insertion, and assessed the effects of mutations on different body size traits compared to the $P$-element-free co-isogenic control. Nearly $60 \%$ of the lines showed significant differences with respect to the control for these traits in one or both sexes and almost $35 \%$ showed trait- and sex-specific
\end{abstract}

effects. Candidate gene mutations frequently increased body size in males and decreased it in females. Among the 92 genes identified, most are involved in development and/or metabolic processes and their molecular functions principally include protein-binding and nucleic acid-binding activities. Although several genes showed pleiotropic effects in relation to body size, few of them were involved in the expression of all traits in one or both sexes. These genes seem to be important for different aspects related to the general functioning of the organism. In general, our results indicate that the genetic architecture of body size traits involves a large fraction of the genome and is largely sex and trait specific.

Heredity (2009) 102, 246-256; doi:10.1038/hdy.2008.117; published online 19 November 2008

Keywords: body size; Drosophila melanogaster, genetic architecture; allometry; sexual dimorphism; $P$-element mutagenesis

\section{Introduction}

Formation of a multicellular organism is a complex highly regulated process that involves differentiation and morphogenesis during development. Organisms differ in size not only between species but there is also considerable variation within species because of both environmental (nutrition, temperature) and genetic factors (Edgar, 2006; Arendt, 2007). Body size is a major fitness-related trait that contributes to successful mate selection, longevity, avoidance of predation and tolerance to heat, cold and starvation among others (for example, Roff, 1992; Schmidt-Nielsen, 1984; Stearns, 1992). Genetic and physiological studies have provided insights into the genetic basis of body size as the target of natural selection (Edgar, 2006; Arendt, 2007). Reproducible differences in body size in natural populations and different environmental factors that affect body size have been extensively studied, particularly, in Drosophila melanogaster (for example, De Jong and Bochdanovits, 2003; De Moed et al., 1999; Partridge et al., 1994; Trotta et al., 2006).

Correspondence: Professor VP Carreira, Departamento de Ecología Genética y Evolución, Facultad de Ciencias Exactas y Naturales, Universidad de Buenos Aires, Ciudad Universitaria, Pabellón II, (C1428EHA), Buenos Aires, Argentina.

E-mail: vpcarreira@ege.fcen.uba.ar

Received 4 April 2008; revised 10 October 2008; accepted 12 October 2008; published online 19 November 2008
Even though the physiology of growth control in insects differs from that of mammals, the genes and signalling pathways involved are similar (Edgar, 2006; Dong et al., 2007; Pan, 2007; Saucedo and Edgar, 2007). The insulin-like growth factor signalling pathway emerges as the principal system that regulates cell proliferation (cell number) and cell growth (cell size) and plays a fundamental role in the determination of metabolism and nutrient storage (Edgar, 2006). An exhaustive genetic dissection has validated the role of this highly conserved pathway in D. melanogaster (for example, Britton et al., 2002; Johnston and Gallant, 2002; Oldham and Hafen, 2003; Saucedo and Edgar, 2002; Shingleton et al., 2005). Converging with the insulin signalling system is the target of rapamycin (TOR) pathway that regulates cell growth rather than cell proliferation (for example, Hennig et al., 2006; Oldham and Hafen, 2003; Wullschleger et al., 2006; Zhang et al., 2000). Particularly, TOR might function as an amino-acid sensor and plays a prominent role in regulation of cell growth in response to nutrients (Cardenas et al., 1999; Zhang et al., 2000). Finally, recent studies have delineated a novel signalling pathway, the Hippo pathway, which has an important role in the regulation of organ size through cell proliferation (for example, Dong et al., 2007; Pan, 2007; Saucedo and Edgar, 2007). Eventually, the adult size of holometabolous insects is determined by the size at which the last-instar larva stops feeding and begins metamorphosis, a process controlled by steroid 
and neuropeptide hormones occurring after a genetically encoded critical weight is achieved (for example, Caldwell et al., 2005; Colombani et al., 2005; Nijhout et al., 2006; Truman et al., 2006). Despite these considerable advances, we still understand little about how size is perceived and how organ-intrinsic size interfaces with whole-body physiology (for example, Caldwell et al., 2005; Conlon and Raff, 1999; Day and Lawrence, 2000; Nijhout, 2003; Nijhout and Emlen, 1998; Shingleton et al., 2007; Stern and Emlen, 1999).

All animal species bear a specific relationship between the size of each organ and overall body size (that is, allometries; Conlon and Raff, 1999; Shingleton et al., 2007). The imaginal discs of insects contain information that specifies their shape and perhaps their approximate size independently of the precise number of cells, but disc growth is also modified both by the overall growth of the animal and by the growth of other discs (for a complete review see Stern and Emlen, 1999). From an evolutionary perspective, adaptations and the emergence of novel phenotypes would require some degree of developmental independence among body parts (Cowley and Atchley, 1990 and references therein). However, different morphological traits cannot be completely independent genetically because of pleiotropy and linkage (Cowley and Atchley, 1990; Nijhout and Emlen, 1998). Traits that derive from the same imaginal discs would be more tightly mutually constrained (Cowley and Atchley, 1990 and references therein). Similarly, traits in males and females would be genetically correlated. As a consequence, a selective response in one sex would entail a shift in phenotype in the other sex which, in turn, implies that only a slight modification of the level of sexual dimorphism would be possible in response to selection (Cowley and Atchley, 1988; Cowley et al., 1986 and references therein). Undoubtedly, substantial progress has been made to elucidate the physiological and environmental factors underpinning differences in body size. However, little is known about the mode of action of the genetic factors (that is, the way in which they interact with each other and the environment) that build and control this trait and its variational properties (that is, its genetic architecture; Hansen, 2006).

Understanding the genetic architecture of complex traits such as body size requires first and foremost identifying the genes that contribute to the expression of the trait, which can be achieved by mutagenesis screens in genetically tractable model organisms such as $D$. melanogaster. Quantitative genetic analysis of subtle effects of $P$-element mutations that have been induced in an isogenic background (Lukacsovich et al., 2001; Bellen et al., 2004) is a highly efficient method for functional genomic analyses (Anholt et al., 1996; Lyman et al., 1996; Norga et al., 2003; Harbison et al., 2004; Sambandan et al., 2006; Mensch et al., 2008).

In this paper, we address the following questions: (1) How many genes are involved in the expression of body size-related traits in D. melanogaster? (2) Which are these genes and what are their principal functions? (3) Does the $P$-element disruption of these genes shows the same effect on different body size-related traits? (4) Does the mutation of these genes present the same effect on males and females? As body size is a complex trait that displays considerable genetic variation in natural populations, our a priori expectation was to find that a large proportion of the genes analysed was involved in body size-related traits expression. The majority of these genes would be related to the general development of the organism and its metabolic functions. However, some of these genes would be involved in more specific functions related to morphogenesis. Therefore, most of these genes would affect all body size-related traits similarly, although some of them would show a local-specific effect. Finally, most of these genes would show a similar effect in both sexes.

In this study, we analysed traits derived from different imaginal discs in both sexes using $P$-element insertion lines. This design allowed us to identify candidate genes that contribute to the expression of body size-related traits in D. melanogaster and to evaluate the implications of genetic correlations between body parts and their sexual dimorphism in the evolution of body size. Candidate gene mutations frequently increased body size in males and decreased it in females. Interestingly, our data indicate that $P$-element insertions in genes that reduced size differences between sexes mapped more frequently at autosomal chromosomes with respect to those that affected both sexes similarly or enlarged sexual dimorphism, which were predominantly X-linked. Among the 92 genes identified, a large proportion is involved in development and/or metabolic processes and their molecular functions principally include protein-binding and nucleic acid-binding activities. In general, our results indicate that the genetic architecture of body size traits involves a large fraction of the genome and is largely sex and trait specific.

\section{Materials and methods}

\section{Drosophila stocks}

We used 191 independent homozygous viable single P[GT1]-element insertion lines, constructed in a coisogenic Canton-S background (Lukacsovich et al., 2001) to identify candidate genes affecting body size-related traits. The lines were kindly provided by Trudy Mackay (North Carolina State University, Raleigh, NC, USA).

$P$-element insertion lines were distributed in nine batches in which 20-25 lines were simultaneously assessed. To account for environmental variation in body size-related traits between batches, 4-8 replicated vials of the control strain (a co-isogenic $P$-element insertion free line with the same genetic background) were run in parallel with each batch. For each line, 300 pairs of sexually mature flies were placed for $8 \mathrm{~h}$ in oviposition chambers. Eggs were allowed to hatch and batches of 30 first-instar larvae were transferred to culture vials containing a standard cornmeal-agar-molasses medium (4 replicates per line). Larvae were raised at $25 \pm 1{ }^{\circ} \mathrm{C}$ and $60-70 \%$ of humidity with a 12:12 light:dark photoperiod until adult emergence.

\section{Body size-related traits}

Four flies of each sex from each vial were randomly chosen and the head, the thorax and the wings of each individual were removed and mounted on slides. Images were captured using a binocular microscope $(\times 10)$ and an attached digital camera connected to a computer. Different morphometric traits were estimated using tpsDig (Rohlf, 2001, version 1.31, available at 
http://life.bio.sunysb.edu/morph/): face width (FW), head width (HW), thorax length (TL) and wing size (WS). FW, which is the smallest distance between the eyes, and HW, which is the distance between the right and the left side of the head capsule (including the eyes), were scored in images with a frontal view of the head. TL, which is the distance between the anterior margin of the thorax and the tip of the scutellum, was scored in images with a lateral (right side up) view of the thorax. For the estimation of WS, 11 landmarks were digitized on the ventral face of the left wing of each fly and a single WS measure (centroid size) was calculated by taking the square root of the sum of squared distances between each landmark and the centroid (the point whose coordinates are the means of the $x$ and $y$ coordinates of all landmarks) of each wing.

\section{Statistical analyses}

Genetic correlation analyses: To include all lines tested in different batches, the values corresponding to each variable were transformed by subtracting from each individual value the mean value of its contemporaneous co-isogenic control line, separately for males and females, and dividing it by the same value. The mean of the transformed values corresponding to each line were used in all correlation analyses. A correlation analysis was performed between each pair of variables within each sex and with each variable between sexes. As we utilized the same values for different correlation analyses, Bonferroni's correction for multiple tests was applied $\left(P_{\mathrm{B}}=0.0125\right)$.

Quantitative genetic analysis: Analysis of variance (ANOVA) was utilized to assess the magnitude of mutational variance for body size-related traits. To include all lines tested in different batches, transformed values were used (see above). Two-way ANOVA was computed for each variable, following the mixed model: $Y=\mu+L+S+L \times S+\varepsilon$, where $\mu$ is the overall mean, $L$ is the random effect of line, $S$ is the fixed effect of sex and $\varepsilon$ represents the error term. Variance components for random sources of variation (line, line by sex interaction and error) were estimated for each variable.

Gene identification and functional analysis: An ANOVA was used to assess the magnitude of the mutational effect induced by $P[G T 1]$ insertions on each body size trait. The one-way ANOVA was computed for each trait, sex and batch separately, following the fixed model: $Y=\mu+L+\varepsilon$, where $L$ is the fixed effect of line and $\varepsilon$ the error variance. To identify which lines were responsible of the significant line factor, phenotypic differences between $P$-element insertion lines and the contemporaneous control line were tested using Dunnett contrasts for each batch. Those lines that exhibited significant differences relative to the control were considered as lines bearing an insertion in a candidate gene.

The number of cases in which size increased or decreased in each sex was subjected to frequency analysis considering all body size-related traits together and each one separately.

To identify the transposon-tagged candidate genes, nucleotide sequences flanking the $P$-element insertion were aligned with corresponding sequences of the reference sequence of the $D$. melanogaster genome.
Table 1 Principal results of the genetic correlation analyses of body size related traits

\begin{tabular}{lcccc}
\hline & \multicolumn{4}{c}{ Males } \\
\cline { 2 - 5 } & Face width & Head width & Thorax length & Wing size \\
\hline Females & & & & \\
Face width & $0.52^{* * *}$ & $0.37^{* * *}$ & $0.44^{* * *}$ & $0.19^{* *}$ \\
Head width & $0.33^{* * *}$ & $0.15^{*}$ a & $0.84^{* * *}$ & $0.59^{* * *}$ \\
Thorax length & $0.49^{* * *}$ & $0.61^{* * *}$ & -0.02 & $0.59^{* * *}$ \\
Wing size & $0.30^{* * *}$ & $0.40^{* * *}$ & $0.58^{* * *}$ & $0.69^{* * *}$ \\
\hline
\end{tabular}

A correlation analysis was performed between each pair of variables within males (above the diagonal), within females (below the diagonal) and between sexes for each variable (on the diagonal). $r$-Value and its significance for each correlation analysis are shown. The mean of the transformed values corresponding to each line were used in all correlation analyses (see text for more details).

${ }^{a}$ Not significant after Bonferroni correction for multiple tests $\left(P_{\mathrm{B}}=0.0125\right.$, see text for more details).

${ }^{*} P<0.05 ;{ }^{* *} P<0.01 ;{ }^{* * *} P<0.001$

Homology searches were performed against release 5 of the published $D$. melanogaster genomic sequence (Flybase, http://flybase.bio.indiana.edu/). Candidate genes were distributed in different gene ontology (GO) terms corresponding to the categories 'biological process' and 'molecular function' according to their annotations (The Gene Ontology Consortium, 2000). This analysis was performed automatically with the aid of the programme FatiGO +, from the Babelomics suite of bioinformatic tools, available at http:/ /www.fatigo.org/ (Al-Shahrour et al., 2006). This programme distributes the genes among the terms corresponding to each category in a non-exclusive manner (that is, a given gene might be associated to more than one GO term).

Pleitropic effects on body size-related traits: Lines were classified according to the degree (number of traits) to which they affected body size-related traits to determine the degree of pleiotropic effects. An ANOVA was performed to assess the magnitude of the mutational effect induced by P[GT1] insertions on body size-related traits in those lines that were the most pleiotropic (that is, those that affected the largest number of traits). The twoway ANOVA was computed with the transformed values of the variables (see above) for each line separately, following the fixed model: $Y=\mu+T+S+T \times S+\varepsilon$, where $T$ is the fixed effect of the trait, $S$ is the fixed effect of the sex and $\varepsilon$ represents the error term. If differences were encountered for all traits in one sex, a one-way ANOVA with trait as fixed factor was performed with the transformed values of the sex showing differences. When the sources of variation trait and/or trait by sex were significant in the analysis corresponding to a line, we identified the means of the traits that were different by means of post hoc comparisons (Tukey's HSD contrasts).

All statistical analyses were performed using the STATISTICA software package (StatSoft Inc., 1999, 2001).

\section{Results}

\section{Genetic correlations analyses}

We performed correlation analyses to assess to what extent different body size traits are correlated in each sex 
separately and between sexes. The correlation between each pair of variables was positive and highly significant in both sexes (Table 1). The percentage of total variance explained by the correlation (the square of $r \times 100$ ) ranged from 9 (FW-WS) to 37\% (HW-TL) in females and from 4 (FW-WS) to $70 \%$ (HW-TL) in males (Table 1). The rank order of the percentage of total variance explained by the correlations between pairs of body size traits is almost identical in both sexes (Table 1). The correlation between sexes for each variable was positive and highly significant only for FW and WS and the percentage of total variance explained by the correlation for these traits was 27 and $47 \%$, respectively (Table 1). Each one of these two body size-related traits showed a greater correlation between sexes than with any other body size-related trait within the same sex. Moreover, the correlation between sexes for HW was not significant after Bonferroni's correction for multiple tests $\left(P_{\mathrm{B}}=0.0125\right.$, Table 1$)$; neither was significant for TL (Table 1).

\begin{tabular}{|c|c|c|c|c|c|}
\hline $\begin{array}{l}\text { Number } \\
\text { of lines } \\
\text { in males }\end{array}$ & $\begin{array}{l}\text { Face } \\
\text { Width }\end{array}$ & $\begin{array}{l}\text { Head } \\
\text { Width }\end{array}$ & $\begin{array}{l}\text { Thorax } \\
\text { Length }\end{array}$ & $\begin{array}{l}\text { Wing } \\
\text { Size }\end{array}$ & $\begin{array}{l}\text { Number } \\
\text { of lines } \\
\text { in females }\end{array}$ \\
\hline 10 & & & & & 7 \\
\hline 3 & & & & & 16 \\
\hline 6 & & & & & 6 \\
\hline $21(3)$ & & & & & $8(3)$ \\
\hline $3(1)$ & & & & & $5(1)$ \\
\hline 1 & & & & & 1 \\
\hline 2 & & & & & 2 \\
\hline 4 & & & & & 5 \\
\hline 2 & & & & & 6 \\
\hline 6 & & & & & 3 \\
\hline 9 & & & & & 2 \\
\hline 2 & & & & & 0 \\
\hline 1 & & & & & 2 \\
\hline $11(3)$ & & & & & $6(3)$ \\
\hline 7 & & & & & 6 \\
\hline
\end{tabular}

Figure 1 Number of lines in which the P-element insertion affected one, two, three or all four body size-related traits in each sex. Numbers between parentheses indicate the lines shared between sexes with a similar effect in body size-related traits affected. Traits affected by the mutation are indicated in dark grey.

\section{Quantitative genetic analysis}

We performed analyses of variance to quantify the effect of genotype and the genotype by sex interaction on variation in body size traits. The results of the ANOVAs indicate that a large number of transposon-tagged genes control the expression of body size-related traits, as the line and the line by sex interaction terms together accounted from 25 to $42 \%$ of the overall phenotypic variance (Table 2). Our analyses showed that $59 \%$ of the lines (113 out of 191 lines tested) differed significantly from the control for at least one trait in either sex (Supplementary information, Table 1). Nearly half of the significant lines (45\% in males and $49 \%$ in females) showed significant differences with respect to the control for only one trait when data were analysed for each sex separately (Figure 1; Supplementary information, Table 1). Most of the lines in which the P-element insertion affected only one trait, showed significant differences from the control line for WS and HW in males and females respectively (Figure 1; Supplementary information, Table 1). Moreover, 18, 23 and 7 lines in males and 22, 10 and 6 lines in females affected two, three and all four traits respectively indicating that the mutated genes have extensive pleiotropic effects on body size traits (Figure 1; Supplementary information, Table 1). Interestingly, only seven $P$-element insertion lines showed similar changes (increasing or decreasing size) in both sexes (numbers between parentheses in Figure 1). In fact, the effect of most gene disruptions on body size traits differed between males and females (Figure 1; Supplementary information, Table 1). The significant line by sex effect (Table 2) suggests that the phenotypic effect of the insertions is sex-biased (Supplementary information, Table 1). Finally, we were surprised to find that, as a consequence of the $P$-element insertion, males showed a significant increase in all traits, whereas in females the scores of the same traits decreased relative to the control line (Table 2). Moreover, this pattern of opposite effects between sexes cannot be attributed to a few lines with major phenotypic effects (Figure 2; Supplementary information, Table 1). A frequency analysis considering all traits together showed that the number of cases in which size increased or decreased in males and females is sex-biased $\left(\chi_{1}^{2}=79.7, P<0.0001\right)$, suggesting that most significant lines exhibited opposite effects between sexes. This tendency, which was highly significant for FW $\left(\chi_{1}^{2}=23.2, P<0.0001\right)$, HW $\left(\chi_{1}^{2}=35.4, P<0.0001\right)$ and TL $\left(\chi_{1}^{2}=43.2, P<0.0001\right)$ but not for WS $\left(\chi_{1}^{2}=0.4, P=0.5286\right)$;

Table 2 Principal results of the ANOVAs for body size-related traits

\begin{tabular}{lcccc}
\hline & d.f. & Face width MS & Head width MS & Wing size MS \\
\hline Line & 190 & $0.05^{* * *}(16)$ & $0.04(4)$ & $0.03(0)$ \\
Sex & 1 & $1.43^{* * *}$ & $0.32^{* *}$ & $1.40^{* * *}$ \\
Line $\times$ sex & 190 & $0.02^{* * *}(9)$ & $0.03^{* * *}(25)$ & $0.02^{* * *}(28)$ \\
Error & a & $0.01(75)$ & $0.01(71)$ & $0.03^{* * * *}(42)$ \\
\hline
\end{tabular}

An ANOVA was performed using the transformed values corresponding to each variable, following a model with line (random) and sex (fixed) as factors (see text for more details). Degrees of freedom (d.f.), mean squares (MS) and the significance of the F-value of each source of variation corresponding to the different ANOVAs are shown. The percentage of the total phenotypic variance explained by each random source of variation corresponding to the different ANOVAs are between parentheses.

For each transformed variable, males showed larger mean values than females.

a $5215,5208,5175$ and 4912 for face width, head width, thorax length and wing size, respectively.

${ }^{* *} P<0.01 ;{ }^{* * *} P<0.001$. 

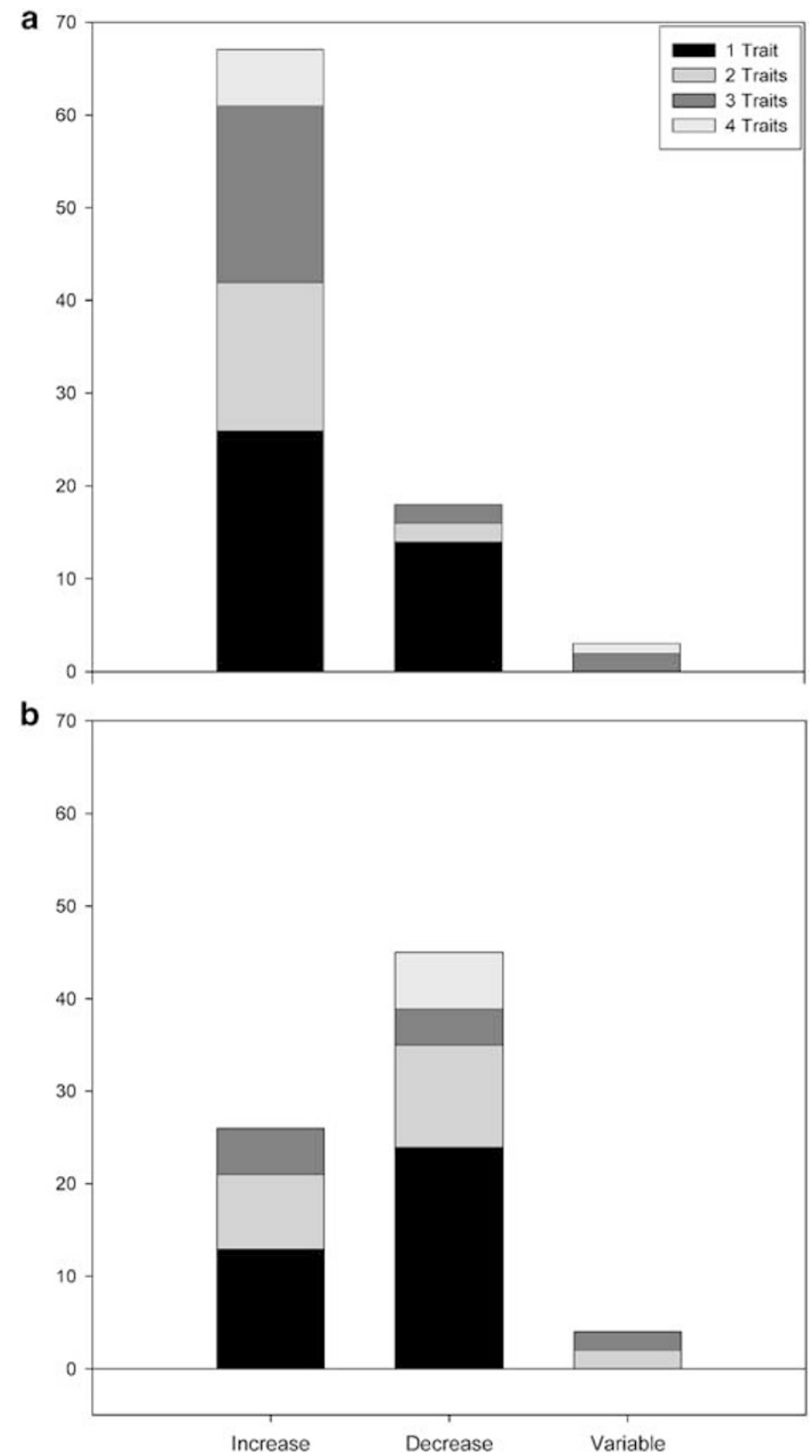

Figure 2 Number of lines in which the mutation increased decreased or increased and decreased the value of one, two, three or all four body size-related traits in males (a) and females (b).

may lead to a less marked sexual dimorphism as a result of $P$-element insertion.

\section{Gene identification and functional analysis}

We identified 92 candidate genes that affect body sizerelated traits based on insertion of the $P$-element within $5 \mathrm{~kb}$ from the transcription initiation site (only the gene nearest to the insertion was selected as candidate gene, except in those cases in which two genes were closer than $1 \mathrm{~kb}$ to the $P$-element insertion site and neither disruption occurred in the gene; Supplementary information, Table 1). An interesting observation was that different insertion sites (that is, different $P$-element insertion lines) in the same gene had different phenotypic effects, indicating that the precise site of the transposon determines its phenotypic effect, as was observed previously for other traits (Rollmann et al., 2006). P-elements inserted in different sites of the first

intron of scalloped (sd) increased HW, TL and WS in both sexes of BG01633 and increased FW in both sexes and WS in males of BG02605 (Supplementary information, Table 1). The mutations-affecting Smrter (Smr) produced an increase in WS in males of BG02219 and a decrease in HW and TL in females and WS in both sexes of BG02262 (Supplementary information, Table 1). These insertions occurred at $375 \mathrm{bp}$ of the $3^{\prime}$ end of the gene and in the first intron of Smr respectively (Supplementary information, Table 1). Mutations-affecting $\beta v$ integrin ( $\beta$ Int- $v$ ) caused a decrease in TL in females of BG01037 and an increase in all traits except WS in males as well as a decrease in HW and TL in females of BG02358 (Supplementary information, Table 1). These insertions took place in the first exon and $699 \mathrm{bp}$ from the $5^{\prime}$ end of $\beta I n t-v$ respectively (Supplementary information, Table 1). Finally, three insertions affected Laminin $A(\operatorname{Lan} A)$, one increased FW in females and decreased WS in males of BG01662, the second increased TL and WS in males of BG02380 and the third caused an increase in all body size parameters except FW in both sexes of BG02469 (Supplementary information, Table 1). These insertions were located at 64, 151 and $170 \mathrm{bp}$ at $5^{\prime}$ side of LanA respectively (Supplementary information, Table 1).

Only two of the 92 candidate genes, forkhead box, subgroup $O$ (foxo) and protein kinase 61C (Pk61C) (Supplementary information, Table 1 ), that affected body size are directly related to pathways that regulate cell proliferation and cell growth, the insulin-like growth factor signalling and TOR pathways. P-element insertion at $104 \mathrm{bp}$ of the $3^{\prime}$ end of foxo, which is related to the negative regulation of cell proliferation and cell size (Kramer et al., 2003; Hwangbo et al., 2004), produced females with reduced HW and TL (Supplementary information, Table 1). Therefore, our results seem to indicate that flies carrying two inactivated copies of foxo present an increased negative regulation of body size in a sex-specific way. Contrary to the activity of foxo, Pk61C mediates positive regulation of cell size (Cho et al., 2001). As the $P$-element insertion at $35 \mathrm{bp}$ of the $3^{\prime}$ end of this gene produced females with reduced HW, TL and WS (Supplementary information, Table 1), this might be a loss-of-function mutation also with a female-specific effect. These results seem to reflect sex-biased constraints in the direction of the effect of loss-of-function mutations.

Genes with significant effects on body size traits were automatically distributed in GO terms corresponding to the categories 'biological process' and 'molecular function' according to their annotations using FatiGO + (AlShahrour et al., 2006). Concerning the biological process, the 56 annotated candidate genes were distributed in 37 categories in a non-exclusive manner (that is, a given gene might be associated to more than one GO term; Table 3). A large proportion of these genes are involved with development and/or metabolic processes (Table 3). Concerning the molecular function, the 71 annotated candidate genes were distributed, in a non-exclusive manner, in 19 categories (Figure 3). Those including more than $10 \%$ of annotated candidate genes are: protein binding, nucleic acid binding, ion binding, transferase activity and nucleotide binding (Figure 3).

We identified those genes that gave rise to the most extreme phenotypes for FW, HW, TL and WS in our panel of candidate genes showing significant effects (Table 4). The percentage of change in the phenotypic 
Table 3 Distribution of candidate genes with annotation in GO terms corresponding to the category 'biological process' using FatiGO+. Genes are distributed in a nonexclusive manner (that is, a given gene might be associated to more than one GO term, see text for more details)

\begin{tabular}{lc}
\hline Biological process GO terms & \% of genes \\
\hline Multicellular organismal development & 64.29 \\
Anatomical structure development & 57.14 \\
Primary metabolic process & 50.00 \\
Cellular developmental process & 50.00 \\
Cellular metabolic process & 48.21 \\
Regulation of biological process & 44.64 \\
Macromolecule metabolic process & 42.86 \\
Cellullar component organization and biogenesis & 33.93 \\
Cell communication & 26.79 \\
Sexual reproduction & 23.21 \\
Establishment of localization & 14.29 \\
Cell cycle & 12.50 \\
Localization of cell & 12.50 \\
Cell adhesion & 12.50 \\
Cell division & 10.71 \\
Behaviour & 8.93 \\
Response to chemical stimulus & 7.14 \\
Regulation of biological quality & 7.14 \\
Biosynthetic process & 7.14 \\
Death & 7.14 \\
Response to stress & 7.14 \\
Chromosome segregation & 5.36 \\
Catabolic process & 5.36 \\
Cell proliferation & 5.36 \\
Response to external stimulus & 3.57 \\
Response to endogenous stimulus & 3.57 \\
Reproductive process & 3.57 \\
Cell recognition & 3.57 \\
Ageing & 3.57 \\
Regulation of molecular function & 3.57 \\
Behavioural interaction between organisms & 1.79 \\
Response to abiotic stimulus & 1.79 \\
Organ growth & 1.79 \\
Multicellular organism growth & 1.79 \\
Protein localization & 1.79 \\
Circulation & 1.79 \\
Response to biotic stimulus & 1.79 \\
\hline
\end{tabular}

The percentage of genes related to each GO term is shown.

value related to the control mean, considering all traits together, varied in females from $11.6 \%$ for increasing FW to $15.4 \%$ for decreasing TL, and in males from 12.2 to $16.7 \%$ from decreasing and increasing TL respectively. There was no case in which a single gene gave rise to the most severe phenotypic effect for all body size traits. The $P$-element insertion in invected (inv), however, was responsible for five out of eight of the most severe decreases in body size observed in both males and females (Table 4).

\section{Pleiotropic effects on body size-related traits}

In 8 of the $191 P$-element lines tested, insertions affected significantly three or four body size traits in both sexes. These are the cases of inv, sd, Doughnut on 2 (dnt) and CG13086, lamina ancestor (lama), CG11550 (the P-element insertion is at $9.6 \mathrm{~kb}$ from the transcription start site of this gene), LanA, CG14782 and lipid storage droplet-2 (Lsd2 ) in which $P$-element insertions occurred in or near the genes (Supplementary information, Table 1). When all lines were ranked according to the magnitude of their effect on each trait and sex separately, these lines were

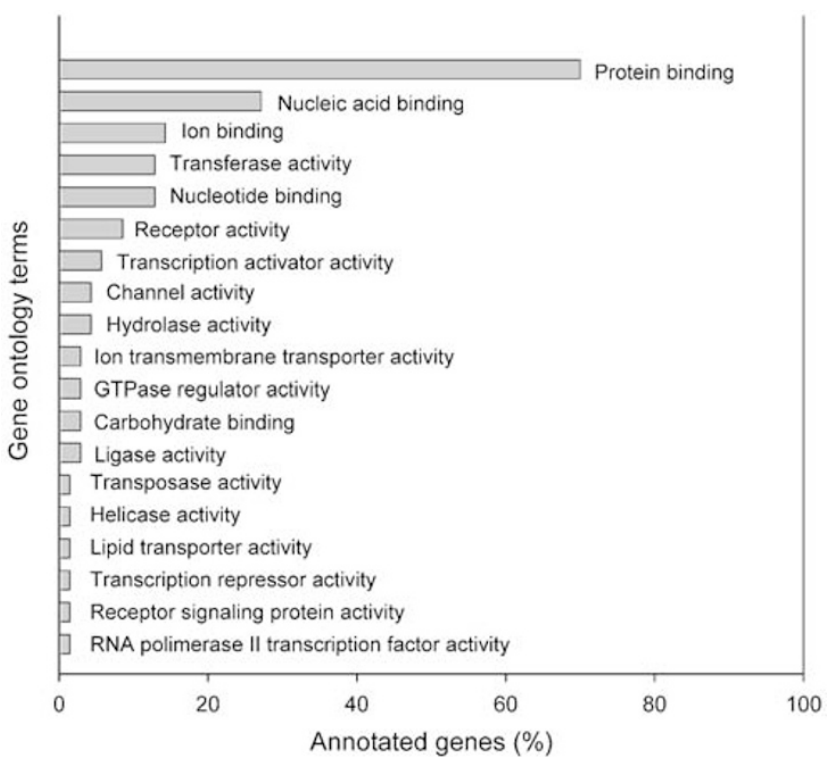

Figure 3 Distribution of candidate genes with annotation in GO terms corresponding to the category 'molecular function' using FatiGO + . Genes are distributed in a non-exclusive manner (that is, a given gene might be associated to more than one GO term, see text for more details).

among those that exhibited the largest decrease (inv, lama, dnt/CG13086, CG11550 and Lsd-2) or the largest increase (sd, dnt/CG13086, LanA, and CG14782) in the scores of most traits.

The principal results of the ANOVAs performed to assess the magnitude of the mutational effect induced by $P$-element insertions on body size traits in those lines that were the most pleiotropic (that is, those that affected the largest number of traits, all four traits in one sex and three in the other) are shown in Table 5. In certain lines, the $P$-element insertion did not have a homogeneous effect neither among traits nor between sexes. For instance, mutation of lama affected different traits in a dissimilar manner, increasing some parts and decreasing others in males and decreasing all of them in females (Table 5; Supplementary information, Table 1). The P-element insertion near CG11550 produced a similar phenotypic effect (Table 5; Supplementary information, Table 1). The insertion near $L s d-2$ represents a different example in which all body size traits were reduced to the same extent in both sexes (Table 5; Supplementary information, Table 1). This mutation caused a mean decrease in body parts of $5.6 \%$, which places this line among the smallest 19 lines. Similarly, mutation of inv decreased size in all traits, although the change was significantly larger in females than males (Table 5; Supplementary information, Table 1). This insertion caused an average decrease in body size of $9 \%$ in males and $12 \%$ in females, which places this line among the top 10 smallest lines.

The principal results of the ANOVAs performed to assess the magnitude of the mutational effect induced by P-element insertions on body size traits in those lines that differed significantly from the control for all traits in one sex (six in males and three in females) are shown in Table 6. The P-element insertion in tropomodulin (tmod; Supplementary information, Table 1) is the only one that 
Table 4 Lines that showed the largest effect for each body size-related trait in males and females

\begin{tabular}{|c|c|c|c|c|c|c|}
\hline \multirow[t]{2}{*}{ Trait } & \multicolumn{3}{|c|}{ Females } & \multicolumn{3}{|c|}{ Males } \\
\hline & Gene & Effect & $\%$ of change & Gene & Effect & $\%$ of change \\
\hline \multirow[t]{2}{*}{ Face width } & lama & - & 13.59 & CG6301 & - & 7.20 \\
\hline & CG12161 & + & 11.57 & CG13130 / bib & + & 15.59 \\
\hline \multirow[t]{2}{*}{ Head width } & $i n v$ & - & 12.88 & $i n v$ & - & 10.31 \\
\hline & BG01635 & + & 1.86 & CG41475 & + & 12.91 \\
\hline \multirow[t]{2}{*}{ Thorax length } & inv & - & 15.41 & inv & - & 12.15 \\
\hline & $s d$ & + & 6.13 & dnt / CG13086 & + & 16.66 \\
\hline \multirow[t]{2}{*}{ Wing size } & $i n v$ & - & 9.88 & $l(1) G 0007$ & - & 7.57 \\
\hline & $s d$ & + & 8.56 & kermit & + & 9.19 \\
\hline
\end{tabular}

The gene/s affected by the P-element insertion and the percentage of change in the phenotypic value (increasing or decreasing size) compared to the phenotype of the control are shown.

${ }^{a}$ No gene could be identified.

Table 5 Principal results of the ANOVAs performed to assess the magnitude of the mutational effect induced by $P$-element insertions on body size-related traits in those lines that were the most pleiotropic

\begin{tabular}{|c|c|c|c|c|}
\hline $\begin{array}{l}\text { Line } \\
\text { Candidate gene }\end{array}$ & $\begin{array}{c}B G 00846 \\
\text { inv }\end{array}$ & $\begin{array}{c}\text { BG02210 } \\
\text { lama }\end{array}$ & $\begin{array}{l}\text { BG02239 } \\
\text { CG11550 }\end{array}$ & $\begin{array}{c}B G 02830 \\
\text { Lsd-2 }\end{array}$ \\
\hline \multicolumn{5}{|l|}{ Source of variation } \\
\hline Trait & $18.07^{* * *}$ & $6.39^{* * *}$ & $3.13^{*}$ & 0.64 \\
\hline Sex & $17.57^{* * *}$ & $328.85^{* * *}$ & $225.02^{* * *}$ & 2.80 \\
\hline Trait $\times$ sex & 0.12 & $42.07^{* * *}$ & $32.37^{* * *}$ & 0.06 \\
\hline \multicolumn{5}{|l|}{ Mean value } \\
\hline \multicolumn{5}{|l|}{ Males } \\
\hline Face width & $-0.05^{\mathrm{a}}$ & $0.01^{\mathrm{a}}$ & $0.08^{\mathrm{a}}$ & -0.06 \\
\hline Head width & $-0.10^{\mathrm{b}}$ & $0.04^{\mathrm{ab}}$ & $0.05^{\mathrm{a}}$ & -0.05 \\
\hline Thorax length & $-0.12^{\mathrm{b}}$ & $0.07^{\mathrm{b}}$ & $0.10^{\mathrm{a}}$ & -0.06 \\
\hline Wing size & $-0.07^{a}$ & $-0.05^{\mathrm{c}}$ & $-0.03^{\mathrm{b}}$ & -0.04 \\
\hline \multicolumn{5}{|l|}{ Females } \\
\hline Face width & $-0.09^{\mathrm{a}}$ & $-0.14^{\mathrm{a}}$ & $-0.07^{\mathrm{a}}$ & -0.07 \\
\hline Head width & $-0.13^{\mathrm{b}}$ & $-0.10^{\mathrm{a}}$ & $-0.09^{\mathrm{ab}}$ & -0.07 \\
\hline Thorax length & $-0.15^{\mathrm{b}}$ & $-0.13^{\mathrm{a}}$ & $-0.14^{\mathrm{b}}$ & -0.07 \\
\hline Wing size & $-0.10^{\mathrm{a}}$ & $-0.05^{\mathrm{b}}$ & $-0.02^{\mathrm{ac}}$ & -0.05 \\
\hline
\end{tabular}

An ANOVA was performed using the transformed values of the variables for each line separately, following a model with trait and sex as fixed factors (see text for more details). F-value and its significance for each source of variation corresponding to the different ANOVAs are shown. Mean values correspond to the average of the transformed value for each trait. Different letters (a, $b, c)$ denote significant differences between mean values in each sex by means of Tukey's HSD contrasts.

${ }^{*} P<0.05 ;{ }^{* * *} P<0.001$.

increased the size of all body parts to the same extent in males (Table 6). Mutations in lines BG01608 and BG01538, where no gene near the insertion sites have been identified and mutations affecting bifocal (bif), longitudinals lacking (lola) and CG8062 increased all body size-related traits in a dissimilar manner in this sex (Table 6; Supplementary information, Table 1). In females, the mutation affecting misshapen (msn) decreased all body size-related traits to the same extent and P-element insertions-affecting CG17836, amnesiac (amn) and CG32529 caused a larger decrease in FW than in any other trait (Table 6; Supplementary information, Table 1). Most of these sex-specific lines were among those lines that showed the largest decrease or increase for any trait and sex. Particularly, the P-element insertion-affecting CG32529 and amn caused a mean size decrease of $5 \%$ in males and $7 \%$ in females.
It is important to note that only inv, lama, CG11550 and Lsd-2 seem to be highly pleiotropic in both sexes and, even though mutations affecting these genes generally caused a decrement in size, there is no apparent pattern in their relative effect on different body size traits. Interestingly, mutations affecting all traits in males caused a size increment in this sex; whereas insertions affecting all traits in females produced a size decrement in this sex. This is a pattern that has been already mentioned for all lines. In these 'pleiotropic sex specific' genes, FW was the trait that showed the greatest phenotypic changes in both sexes. In fact, this trait showed a mean enlargement of $8.7 \%$ in males with respect to the control line (the average increases for $\mathrm{HW}$, TL and WS were 6.8, 7.8 and $3.4 \%$ respectively) whereas in females FW decreased, on average, 9.3\% with respect to the control (the average decreases for HW, TL and WS were $5.6,5.8$ and $6.2 \%$ respectively).

\section{Discussion}

We have used lines obtained by $P$-element insertional mutagenesis (Lukacsovich et al., 2001) to identify candidate genes involved in the determination of four body size-related traits: FW, HW, TL and WS. Although the number of genes affected by $P$-element insertions only represents $1-1.5 \%$ of all the genes in the Drosophila genome, almost $60 \%$ affected at least one body sizerelated trait in one or both sexes and nearly $35 \%$ affected only one trait in one sex. These results reflect the complexity of the genetic architecture of these traits, involving a large portion of the genome in the determination of body size-related phenotypes examined.

One interesting feature of our results is that, on average, the effects of $P$-element insertions tended to diminish the sexual dimorphism in body size. Among the 92 genes identified, a large proportion is involved in development and/or metabolic processes and their molecular functions principally include protein-binding and nucleic acid-binding activities. Some of these genes that orchestrate the expression of body size-related traits in one or both sexes, also contribute to other adaptively important traits such as abdominal and sternopleural bristle number (Norga et al., 2003), starvation resistance (Harbison et al., 2004) and olfactory behaviour (Sambandan et al., 2006, Supplementary information, Table 1). In general, our results indicate that the genetic architecture 
Table 6 Principal results of the ANOVAs performed to assess the magnitude of the mutational effect induced by $P$-element insertions on body size related traits in those lines that differed from the control line for all size related traits in one sex

\begin{tabular}{|c|c|c|c|c|c|}
\hline \multirow[t]{2}{*}{ Lines (candidate genes) } & \multicolumn{5}{|c|}{ Mean value } \\
\hline & $F$ (trait) & Face width & Head width & Thorax length & Wing size \\
\hline \multicolumn{6}{|l|}{ Males } \\
\hline BG01538 $\S$ & $9.86^{* * *}$ & $0.08^{\mathrm{a}}$ & $0.10^{\mathrm{a}}$ & $0.12^{\mathrm{a}}$ & $0.03^{\mathrm{b}}$ \\
\hline BG01608 $\S$ & $8.17^{* * *}$ & $0.09^{\mathrm{a}}$ & $0.04^{\mathrm{b}}$ & $0.06^{\mathrm{b}}$ & $0.04^{\mathrm{b}}$ \\
\hline BG01637 (tmod) & 1.46 & 0.06 & 0.04 & 0.05 & 0.03 \\
\hline BG02056 (lola) & $3.17^{*}$ & $0.08^{\mathrm{a}}$ & $0.05^{\mathrm{ab}}$ & $0.05^{\mathrm{ab}}$ & $0.04^{\mathrm{b}}$ \\
\hline BG02241 (bif) & $13.55^{* * *}$ & $0.11^{\mathrm{a}}$ & $0.06^{\mathrm{b}}$ & $0.06^{\mathrm{b}}$ & $0.04^{\mathrm{b}}$ \\
\hline BG02412 (CG8062) & $21.84^{* * *}$ & $0.10^{\mathrm{a}}$ & $0.13^{\mathrm{a}}$ & $0.13^{\mathrm{a}}$ & $0.03^{b}$ \\
\hline \multicolumn{6}{|l|}{ Females } \\
\hline BG01011 (msn) & 0.61 & -0.07 & -0.07 & -0.05 & -0.08 \\
\hline BG01279 (CG17836) & $7.06^{* * *}$ & $-0.10^{\mathrm{a}}$ & $-0.04^{\mathrm{b}}$ & $-0.06^{\mathrm{b}}$ & $-0.06^{\mathrm{b}}$ \\
\hline BG02286 (amn/CG32529) & $4.72^{* *}$ & $-0.11^{\mathrm{a}}$ & $-0.06^{b}$ & $-0.06^{b}$ & $-0.06^{b}$ \\
\hline
\end{tabular}

An ANOVA was performed using the transformed values of the variables for each line separately, following a model with trait as fixed factor (see text for more details). F-value and its significance for each source of variation corresponding to the different ANOVAs are shown. Mean values correspond to the average of the transformed value for each trait. Different letters ( $a, b)$ denote significant differences between mean values by means of Tukey HSD contrasts.

${ }^{*} P<0.05 ;{ }^{* *} P<0.01 ;{ }^{* * *} P<0.001$.

$\S$ No gene could be identified.

of body size traits involves a large fraction of the genome and is largely sex and trait specific.

Genetic correlations between body size-related traits are well documented in Diptera (for example, Cowley and Atchley, 1988, 1990; David et al., 2006; De Moed et al., 1997; Loeschcke et al., 1999; Misra, 1966; Moreteau et al., 2003; Norry et al., 1997; Rohlf and Sokal, 1972, Scheiner et al., 1991; Sisodia and Singh, 2001). Most studies reported that correlations among measurements of different body parts are usually positive indicating that cell proliferation and cell growth rates of different imaginal discs may be proportional during larval growth, providing a possible explanation to the apparent constraints that limit divergence from the fundamental body plan of flies. However, in our study, the percentage of total variance explained by correlations among traits was generally lower than previously reported in D. melanogaster (for example, Cowley and Atchley, 1988, 1990; De Moed et al., 1997; Scheiner et al., 1991). Furthermore, our results do not entirely support the hypothesis that traits derived from the same imaginal disc are more correlated than those derived from different precursors as it was reported before (Cowley and Atchley, 1990). Certainly, neither traits derived from the wing disc (thorax and wing traits) nor from the eyeantennal disc (head traits) showed greater correlation coefficients between them than between traits derived from different embryonic precursors. For instance, FW and HW were more correlated with TL than between themselves and, even tough WS was correlated with TL, the correlation coefficient between HW and TL was larger. Thus, our results support an autonomous growth model for different body parts, as proposed by Stern and Emlen (1999), who argued that the growth of different structures might be partially regulated by mechanisms involving either a centralized system for translating body size into a growth signal, and/or by communication between imaginal tissues and the rest of the body. However, if different organs vary in the density of receptors for such a 'body size signal', this would lead to differences in the 'sensitivity' of each organ, which might lead to organ-specific allometries. Such a mechanism might underlie genetic variation within populations and account for sexual dimorphism (Stern and Emlen, 1999). In this sense, FW and WS were the only traits that were positively (and significantly) correlated across sexes. This result suggests a sexual dimorphism in the genetic architecture of some body size traits, which might be a common feature with others traits such as starvation resistance (Harbison et al., 2004), abdominal and sternopleural bristle number (Norga et al., 2003) and olfactory behaviour (Anholt et al., 2003; Sambandan et al., 2006).

Our quantitative genetic analysis revealed extensive genetic variation for all body size traits studied, although for two traits it was masked by strong line by sex interactions. The relative percentages of phenotypic variation explained by genotype (line) and genotype by sex (line by sex) interaction also reveal important aspects of the genetic architecture of body size traits. First, the percentages of total phenotypic variance explained by both sources of variation for different body size traits $(25-42 \%)$ suggest that several genes contribute to the expression of these traits. Furthermore, the effects of these genes depend on the sex because a considerable percentage of total phenotypic variance for different body size-related traits can be accounted for by the line by sex interaction (8-42\%). Second, and perhaps more surprising, is that the percentages of phenotypic variance explained by the line and line by sex components of variance for body size traits are among the lowest values recorded so far as compared to other quantitative traits studied using the same suite of co-isogenic P-element insertion lines: $81 \%$ for starvation resistance (Harbison et al., 2004), 64\% for developmental time (Mensch et al., 2008), 57\% for sternopleural bristle number (Norga et al., 2003), 56\% for abdominal bristle number (Norga et al., 2003) and $31 \%$ for adult olfactory behaviour (Sambandan et al., 2006). However, it must be considered that these results were obtained using a particular isogenic background. Furthermore, body size-related traits analysed are associated to certain measurement error that, in combination with 
the inter-individual variance, might greatly reduce the proportion of phenotypic variance explained by the genetic terms.

Further, half of the lines analysed for each sex separately showed differences for only one trait that explains the low percentage of total variance explained by the correlation between size traits in each sex. Except for seven lines in which the mutation affected body size traits similarly in both sexes, mutations generally increased the size of one or more traits in males whereas they decreased the value of one or more traits in females. This opposite effect between sexes, which reduces sexual dimorphism for body size, explains the low percentage of total variance explained by the correlation between sexes for each body size trait. However, these opposite effects between sexes caused by $P$-element insertions were significant for head traits (HW and FW) and TL but not for WS. These results give strong support to our hypothesis that the genetic architectures of body sizerelated traits (at least for FW, HW and TL) differ between males and females and may reflect evolutionary limitations on the divergence for these traits between the sexes. Particularly, the reduction of sexual dimorphism as a consequence of the $P$-element insertional mutations suggests that body size in each sex might be subjected to different selective forces in nature that, in turn, might lead to the differences between sexes.

This is in line with previous studies (Fisher, 1958; Lande, 1980), concerning the evolution of sexual dimorphism that stated that, by default, genes would be expressed in both sexes (that is, traits in males and females would be genetically correlated). As a consequence, a selective response in one sex would entail a shift in phenotype in the other sex. In the case where males and females are selected for different trait values, a move toward the optimum of one sex would move the other sex away from its optimum. In this case, mutations would be 'sexually antagonistic' because their positive effect on one sex would be associated with a negative effect in the other sex. The fact that sexually antagonistic alleles segregate in the population might select for a breakdown of the genetic correlation between the sexes, for example through the action of modifiers (Rice, 1984). In this sense, Rice (1984) pointed out that genes coding for sexually dimorphic traits should be located disproportionately on the $X$ chromosome. However, our data indicate that $P$-element insertions in genes that reduced size differences between sexes, mapped more frequently at autosomal chromosomes respect to those that affected both sexes similarly or enlarged sexual dimorphism, which were predominantly X-linked $\left(\chi_{1}^{2}=12.22\right.$, $P=0.0005)$. It is interesting to note that the distribution of genes affecting body size traits through the genome did not depart from a random one $\left(\chi_{2}^{2}=0.05, P=0.9753\right)$. This result is apparently in contradiction with previous studies that reported an increased contribution of the $X$ chromosome to genetic variance for body sizerelated traits in D. melanogaster (Kearsey and Kojima, 1967; Cowley and Atchley, 1988). Finally, sex-specific effects on body size-related traits might imply the existence of strong interactions between genes involved with the genetic cascade responsible of the genetical sex and many other genes (genes directly related to morphogenesis and other type of genes) all over the genome. This, in turn, suggests that the genetic architecture of sexual dimorphism for body size traits is very complex, involving interactions among different genetic networks.

The functional analysis of the 92 candidate genes revealed that half of them are involved with development and/or metabolic processes. Furthermore, 70\% of the genes present protein-binding activity and more than $27 \%$ nucleic acid-binding capability.

Of the 92 genes, 8 affect three or four body size traits in both sexes (dnt, inv, LanA, lama, CG13086, sd, CG14782 and $L s d-2)$; at least four contribute to the expression of all traits in males (lola, tmod, bif and CG8062), whereas four affect the expression of all traits in females ( $m s n$, CG17836, CG32529 and amn). Mutations in four of these genes (inv, Lsd-2, amn and CG32529) resulted in large decreases in almost all body size traits in both sexes. In contrast, only taking together both $P$-element insertions affecting $s d$, we observed an increase in all body sizerelated traits.

As far as we know, there are no records relating most of these pleiotropic genes with body or organ size (Flybase release 5). On one side, inv is closely related in genome position, sequence and pattern of expression to engrailed (a segment polarity gene; Coleman et al., 1987). On the other side, $L s d-2$ is related to the transportation of lipid particles along the microtubules and its regulation (Welte et al., 2005) as well as to lipid (Gronke et al., 2003) and triacylglycerol sequestering (Teixeira et al., 2003). In general, studies of the effect of Lsd-2 lack-of-function and gain-of-function mutations have shown that this gene is essential for an adjustment of the organismal lipid storage level. In contrast, amn has been reported to be involved in the regulation of body size. Recent studies in Drosophila showed that the activity of insulin-signalling pathway in the prothoracic gland modulates ecdysone release regulating both the duration and rate of larval growth (Caldwell et al., 2005; Colombani et al., 2005). In this context, experimental evidence raised the possibility that an amn neuropeptide might actively participate in regulating ecdysone synthesis (Caldwell et al., 2005). According to this hypothesis, the mutation of amn might lead to a premature ecdysone release leading to the development of small adults. Our results are in line with this view as the mutation of amn caused a significant reduction of body size-related traits. Finally, sd has been related mostly to the imaginal disc-derived wing morphogenesis (Dworkin and Gibson, 2006). Viable mutants of $s d$ exhibit defects that can include gapping of the wing margin and ectopic bristle formation on the wing (Campbell et al., 1991). However, contrarily to the cases mentioned above, $s d$ mutation increased the size of all traits studied although this results from taking into account the phenotypic effects of two independent mutants. Apparently, different insertions in this gene affected body size traits in a dissimilar fashion, suggesting that the precise location of the transposon determines the phenotypic effect, as was observed previously for other traits (Rollmann et al., 2006). Therefore, even though these pleiotropic genes apparently play a role in body size determination, it seems that they have barely been associated to morphogenesis. An inspection of the GO terms corresponding to the categories 'molecular function' and 'biological process' related to these genes (Supplementary information, Table 2) suggests that they are important for different aspects related to the general functioning of the organism which, in turn, 
implies that their mutation might cause a general adverse effect on growth. However, this effect was not observed in the case of $s d$ mutations. According to this idea, P-element insertions-affecting inv, Lsd-2 and amn enlarged egg-to-adult developmental time but this trait was not modified when $s d$ was mutated (Mensch et al., 2008)

In general terms, our results support the idea that the genetic architecture of body size is complex with different genes contributing to different traits in a sexually dimorphic manner. Particularly, its largely polygenic basis is composed by genes related to the general development of the organism and its metabolic functions as well as genes associated to more specific functions related to the expression of body size-related traits. Further studies of the developmental stage or organ in which these genes are expressed will help to elucidate the relevance of these genes on the regulation of the size of different body structures.

\section{Acknowledgements}

We thank E Hasson, IM Soto, RRH Anholt and TFC Mackay for comments and suggestions that helped to improve this paper. We also thank three anonymous reviewers for constructive criticisms on an earlier version of this paper. This work was supported by grants of the Universidad de Buenos Aires, Consejo Nacional de Investigaciones Científicas y Técnicas (CONICET) and Agencia Nacional de Promoción Científica y Técnica. VP Carreira and J Mensch are recipients of postgraduate scholarships of CONICET. JJ Fanara is member of Carrera del Investigador Científico (CONICET).

\section{References}

Al-Shahrour F, Minguez P, Tárraga J, Montaner D, Alloza E, Vaquerizas JMM et al. (2006). BABELOMICS: a systems biology perspective in the functional annotation of genomescale experiments. Nucleic Acids Res 34: 472-476.

Anholt RRH, Dilda CL, Chang S, Fanara JJ, Kulkarni NH, Ganguly I et al. (2003). The genetic architecture of odorguided behavior in Drosophila: epistasis and the transcriptome. Nat Genet 35: 180-184.

Anholt RRH, Lyman RF, Mackay TFC (1996). Effects of single P-element insertions on olfactory behavior in Drosophila melanogaster. Genetics 143: 293-301.

Arendt J (2007). Ecological correlates of body size in relation to cell size and cell number: patterns in flies, fish, fruits and foliage. Biol Rev Camb Philos Soc 82: 241-256.

Bellen HJ, Levis RW, Liao G, He Y, Carlson JW, Tsang G et al. (2004). The BDGP gene disruption project: single transposon insertions associated with $40 \%$ of Drosophila genes. Genetics 167: 761-781.

Britton JS, Lockwood WK, Li L, Cohen SM, Edgar BA (2002). Drosophila's insulin/PI3-kinase pathway coordinates cellular metabolism with nutritional conditions. Dev Cell 2: 239-249.

Caldwell PE, Walkiewicz M, Stern M (2005). Ras activity in the Drosophila prothoracic gland regulates body size and developmental rate via ecdysone release. Curr Biol 15: 1785-1795.

Campbell SD, Duttaroy A, Katzen AL, Chovnick A (1991). Cloning and characterization of the scalloped region of Drosophila melanogaster. Genetics 127: 367-380.

Cardenas ME, Cutler NS, Lorenz MC, Di Como CJ, Heitman J (1999). The TOR signaling cascade regulates gene expression in response to nutrients. Genes Dev 13: 3271-3279.

Coleman KG, Poole SJ, Weir MP, Soeller WC, Kornberg T (1987). The invected gene of Drosophila: sequence analysis and expression studies reveal a close kinship to the engrailed gene. Genes Dev 1: 19-28.

Colombani J, Bianchini L, Layalle S, Pondeville E, DauphinVillemant C, Antoniewski C et al. (2005). Antagonistic actions of ecdysone and insulins determine final size in Drosophila. Science 310: 667-670.

Conlon I, Raff M (1999). Size control in animal development. Cell 96: 235-244.

Cowley DE, Atchley WR, Rutledge JJ (1986). Quantitative genetics of Drosophila melanogaster. I. Sexual dimorphism in genetic parameters for wing traits. Genetics 114: 549-566.

Cowley DE, Atchley WR (1990). Development and quantitative genetics of correlation structure among body parts of Drosophila melanogaster. Am Nat 135: 242-268.

Cowley DE, Atchley WR (1988). Quantitative genetics of Drosophila melanogaster. II. Heritabilities and genetic correlations between sexes for head and thorax traits. Genetics 119: 421-433.

Cho KS, Lee JH, Kim S, Kim D, Koh H, Lee J et al. (2001). Drosophila phosphoinositide-dependent kinase-1 regulates apoptosis and growth via the phosphoinositide 3-kinasedependent signaling pathway. Proc Natl Acad Sci USA 98: 6144-6149.

David JR, Araripe LO, Bitner-Mathé BC, Capy P, Goñi B, Klaczko LB et al. (2006). Quantitative trait analysis and geographic variability of natural populations of Zaprionus indianus, a recent invader in Brazil. Heredity 96: 53-62.

Day SJ, Lawrence PA (2000). Measuring dimensions: the regulation of size and shape. Development 127: 2977-2987.

De Jong G, Bochdanovits Z (2003). Latitudinal clines in Drosophila melanogaster: body size, allozyme frequencies, inversion frequencies, and the insulin-signalling pathway. I Genet 82: 207-223.

De Moed GH, De Jong G, Scharloo W (1997). Environmental effects on body size variation in Drosophila melanogaster and its cellular basis. Genet Res 70: 35-43.

De Moed GH, Kruitwagen CLJJ, De Jong G, Scharloo W (1999). Critical weight for the induction of pupariation in Drosophila melanogaster: genetic and environmental variation. J Evol Biol 12: 852-858.

Dong J, Feldmann G, Huang J, Wu S, Zhang N, Comerford SA et al. (2007). Elucidation of a universal size-control mechanism in Drosophila and mammals. Cell 130: 1120-1133.

Dworkin I, Gibson G (2006). Epidermal growth factor receptor and transforming growth factor-beta signaling contributes to variation for wing shape in Drosophila melanogaster. Genetics 173: 1417-1431.

Edgar BA (2006). How flies get their size: genetics meets physiology. Nat Rev Genet 7: 907-916.

Fisher RA (1958). The Genetical Theory of Natural Selection. Dover Publications: New York.

Gronke S, Beller M, Fellert S, Ramakrishnan H, Jackle H, Kuhnlein RP (2003). Control of fat storage by a Drosophila PAT domain protein. Curr Biol 13: 603-606.

Hansen TF (2006). The evolution of genetic architecture. Annu Rev Ecol Evol Syst 37: 123-157.

Harbison ST, Yamamoto AH, Fanara JJ, Norga KK, Mackay TFC (2004). Quantitative trait loci affecting starvation resistance in Drosophila melanogaster. Genetics 166: 1807-1823.

Hennig KM, Colombani J, Neufeld TP (2006). TOR coordinates bulk and targeted endocytosis in the Drosophila melanogaster fat body to regulate cell growth. J Cell Biol 173: 963-974.

Hwangbo DS, Gersham B, Tu MP, Palmer M, Tatar M (2004). Drosophila dFOXO controls lifespan and regulates insulin signalling in brain and fat body. Nature 429: 562-566.

Johnston LA, Gallant P (2002). Control of growth and organ size in Drosophila. Bioessays 24: 54-64.

Kearsey MJ, Kojima K. (1967). The genetic architecture of body weight and egg hatchability in Drosophila malanogaster. Genetics 56: 23-37. 
Kramer JM, Davidge JT, Lockyer JM, Staveley BE (2003). Expression of Drosophila FOXO regulates growth and can phenocopy starvation. BMC Dev Biol 3: 5-18.

Lande R (1980). Sexual dimorphism, sexual selection, and adaptation in polygenic characters. Evolution 34: 292-305.

Loeschcke V, Bundgaard J, Barker JSF (1999). Reaction norms across genetic parameters at different temperatures for thorax and wing size traits in Drosophila aldrichi and D. buzzatii. J Evol Biol 12: 605-623.

Lukacsovich T, Asztalos Z, Awano W, Baba K, Kondo S, Niwa S et al. (2001). Dual-tagging gene trap of novel genes in Drosophila melanogaster. Genetics 157: 727-742.

Lyman RF, Lawrence F, Nuzhdin SV, Mackay TFC (1996). Effects of single $P$-element insertions on bristle number and viability in Drosophila melanogaster. Genetics 143: 277-292.

Mensch J, Lavagnino N, Carreira VP, Massaldi A, Hasson E, Fanara JJ. 2008. Identifying candidate genes affecting developmental time in Drosophila melanogaster: pervasive pleiotropy and gene-by-environment interaction. BMC Dev Biol 8: 78 .

Misra RK (1966). Vectorial analysis for genetic clines in body dimensions in populations of Drosophila subobscura Coll. and comparison with those of D. robusta Sturt. Biometrics 22 469-487.

Moreteau B, Gibert P, Pétavy JC, Huey RB, David JR (2003). Morphometrical evolution in a Drosophila clade: the Drosophila obscura group. I Zool Syst Evol Res 41: 64-71.

Nijhout HF, Davidowitz G, Roff DA (2006). A quantitative analysis of the mechanism that controls body size in Manduca sexta. J Biol 5: 16-30.

Nijhout HF, Emlen DJ (1998). Competition among body parts in the development and evolution of insect morphology. Proc Natl Acad Sci USA 95: 3685-3689.

Nijhout HF (2003). The control of body size in insects. Dev Biol 261: 1-9.

Norga KK, Gurganus MC, Dilda CL, Yamamoto A, Lyman RF, Patel PH et al. (2003). Quantitative analysis of bristle number in Drosophila mutants identifies genes involved in neural development. Curr Biol 13: 1388-1397.

Norry FM, Vilardi JC, Hasson E (1997). Genetic and phenotypic correlations among size-related traits, and heritability variation between body parts in Drosophila buzzatii. Genetica 101: 131-139.

Oldham S, Hafen E (2003). Insulin/IGF and target of rapamycin signaling: a TOR de force in growth control. Trends Cell Biol 13: 79-85.

Pan D (2007). Hippo signaling in organ size control. Genes Dev 2: 886-897.

Partridge L, Barrie B, Fowler K, French V (1994). Evolution and development of body size and cell size in Drosophila melanogaster in response to temperature. Evolution 48: 1269-1276.

Rice WR (1984). Sex chromosomes and the evolution of sexual dimorphism. Evolution 38: 735-742.

Roff DA (1992). The Evolution of Life Histories. Chapman and Hall: New York.

Rohlf FJ (2001). tpsDig Free software available at http:/ / www.morph.bio.sunysb.edu/morph/index.html.
Release 1.40. Department of Ecology and Evolution, State University of New York: New York.

Rohlf FJ, Sokal RR (1972). Comparative morphometrics by factor analysis in two species of Diptera. Z Morphol Tiere 72: 36-45.

Rollmann SM, Magwire MM, Morgan TJ, Ozsoy ED, Yamamoto A, Mackay TFC et al. (2006). Pleiotropic fitness effects of the Tre1-Gr5a region in Drosophila melanogaster. Nat Genet 38: 824-829.

Sambandan D, Yamamoto A, Fanara JJ, Mackay TFC, Anholt RR (2006). Dynamic genetic interactions determine odor-guided behavior in Drosophila melanogaster. Genetics 174: 1349-1363.

Saucedo LJ, Edgar BA (2002). Why size matters: altering cell size. Curr Opin Genet Dev 12: 565-571.

Saucedo LJ, Edgar BA (2007). Filling out the Hippo pathway. Nat Rev Mol Cell Biol 8: 613-621.

Scheiner SM, Caplan RL, Lyman RF (1991). The genetics of phenotypic plasticity. III. Genetic correlations and fluctuating aymmetries. J Evol Biol 4: 51-68.

Schmidt-Nielsen K (1984). Scaling: Why is animal size so important?. Cambridge University Press: Cambridge.

Shingleton AW, Das J, Vinicius L, Stern DL (2005). The temporal requirements for insulin signaling during development in Drosophila. PloS Biol 3: e289.

Shingleton AW, Frankino WA, Flatt T, Nijhout HF, Emlen DJ (2007). Size and shape: the developmental regulation of static allometry in insects. Bioessays 29: 536-548.

Sisodia S, Singh BN (2001). Mating success and morphometric traits in Drosophila ananassae. Curr Sci 80: 1444-1447.

StatSoft Inc (1999). STATISTICA. A Data Analysis Software System. http:/ / www.statsoft.com.

StatSoft Inc (2001). STATISTICA. A Data Analysis Software System Release 6.0. Tulsa, OKhttp://www.statsoft.com

Stearns SC (1992). The Evolution of Life Histories. Oxford University Press: Oxford

Stern DL, Emlen DJ (1999). The developmental basis for allometry in insects. Development 126: 1091-1101.

Teixeira L, Rabouille C, Rorth P, Ephrussi A, Vanzo NF (2003). Drosophila perilipin/ADRP homologue Lsd2 regulates lipid metabolism. Mech Dev 120: 1071-1081.

The Gene Ontology Consortium (2000). Gene Ontology: tool for the unification of biology. Nat Genet 25: 25-29.

Trotta V, Calboli FCF, Ziosi M, Guerra D, Pezzoli MC, David JR et al. (2006). Thermal plasticity in Drosophila melanogaster: a comparison of geographic populations. BMC Evol Biol 6: $67-79$.

Truman JW, Hiruma K, Allee JP, MacWhinnie SGB, Champlin DT, Riddiford LM (2006). Juvenile hormone is required to couple imaginal disc formation with nutrition in insects. Science 312: 1385-1388.

Welte MA, Cermelli S, Griner J., Viera A, Guo Y, Kim DH et al. (2005). Regulation of lipid-droplet transport by the perilipin homolog LSD2. Curr Biol 15: 1266-1275.

Wullschleger S, Loewith R, Hall MN (2006). TOR signaling in growth and metabolism. Cell 124: 471-484.

Zhang HB, Stallock JP, Ng JC, Reinhard C, Neufeld TP (2000). Regulation of cellular growth by the Drosophila target of rapamycin dTOR. Genes Dev 14: 2712-2724.

Supplementary Information accompanies the paper on Heredity website (http://www.nature.com/hdy) 